\title{
Effect of compensatory acceleration training in combination with accommodating resistance on upper body strength in collegiate athletes
}

This article was published in the following Dove Press journal:

Open Access Journal of Sports Medicine

4 August 2014

Number of times this article has been viewed

\section{Margaret $\mathrm{T}$ Jones}

Sports Medicine Assessment, Rehabilitation, and Testing Laboratory, School of Recreation, Health, and Tourism, George Mason University, Manassas, VA, USA
Correspondence: Margaret T Jones Sports Medicine Assessment, Rehabilitation, and Testing Laboratory, School of Recreation, Health, and Tourism, George Mason University, 10900 University Blvd, MS 4E5, Manassas, VA 20II 0-2203, USA

Tel +I 7039933247

Fax +I 7039932025

EmailmjonesI5@gmu.edu
Purpose: To determine the impact of inclusion of a band or chain compensatory acceleration training (CAT), in a 5-week training phase, on maximal upper body strength during a 14-week off-season strength and conditioning program for collegiate male athletes.

Patients and methods: Twenty-four National Collegiate Athletic Association (NCAA) collegiate baseball players, who were familiar with the current strength and conditioning program and had a minimum of 1 year of formal collegiate strength and conditioning experience, participated in this off-season training study. None of the men had participated in CAT before. Subjects were matched following a maximal effort (1-repetition maximum [1-RM]) bench press test in week 1, then were randomly assigned into a band-based CAT group or a chain-based CAT group and participated in a 5 -week training phase that included bench pressing twice per week. Upper body strength was measured by 1 -RM bench press again at week 6 . A $2 \times 2$ mixed factorial (method $\times$ time) analysis of variance was calculated to compare differences across groups. The alpha level was set at $P<0.05$.

Results: No difference $\left(F_{1,22}=0.04, P=0.84\right)$ existed between the band-based CAT and chainbased CAT groups. A significant difference was observed between pre- and posttests of 1-RM bench $\left(F_{1,22}=88.46, P=0.001\right)$.

Conclusion: A 5-week band CAT or chain CAT training program used in conjunction with an off-season strength and conditioning program can increase maximal upper body strength in collegiate baseball athletes. Using band CAT and/or chain CAT as a training modality in the off-season will vary the training stimulus from the traditional and likely help to maintain the athlete's interest.

Keywords: variable resistance, band, baseball, chain, resistance training

\section{Introduction}

Successful skill execution in baseball often requires the development of force over a short period of time. Strength is critical to developing force rapidly and is integral to the baseball skills of batting, throwing, and running. Consistent strength training has been shown to lead to an increased throwing velocity ${ }^{1,2}$ and bat swing speed ${ }^{3,4}$ in baseball athletes. The importance of strength, power, and agility, in relation to batting and fielding performance, has been demonstrated, ${ }^{5,6}$ and it has been recommended that their development be the focus of off-season strength and conditioning training programs. $^{7}$

Upper body strength is believed to be important in baseball, and as such, the bench press is commonly included in resistance training programs for baseball athletes. ${ }^{1,3,8,9}$ The Olympic/ "free bar" bench press is considered to be an optimal exercise for developing 
upper body musculature, and a variety of bench press methods have been included in off-season training programs, with the goal of improving maximal upper body strength. ${ }^{8,10-12}$

Compensatory acceleration training (CAT), which is the process of attempting to achieve maximal acceleration with a moderate to high load, ${ }^{13,14}$ has been shown to be effective for increasing maximal upper body strength. ${ }^{15}$ This training technique, also referred to as intended maximal concentric acceleration (IMCA), has been used by powerlifters, ${ }^{14,16-18}$ football players, ${ }^{15}$ and baseball athletes ${ }^{19}$ for lower- and upper-body muscular strength and power development. The ability to complete the same amount of work in a shorter time frame leads to an increased rate of force development, ${ }^{12}$ which is essential to a power sport like baseball. When using CAT or IMCA training, it is recommended that the athlete receives verbal instruction to move the weight quickly as the exercise is performed. ${ }^{15-19}$

Accommodating resistance (AR) or variable resistance training methods permit the weightlifter to maintain movement velocity throughout the complete range of motion, by providing decreased total resistance at the point of weakest leverage for the muscle joint or "sticking point" within the exercise. ${ }^{10,20}$ Training with AR methods, such as elastic bands and weighted chains, will minimize the amount of deceleration at the end of the concentric portion of the lifting exercise, thereby permitting an extended period of acceleration and an improved rate of force development. ${ }^{12,16-18,21}$ Additionally, performing the bench press with AR is thought to minimize stress, due to the variable resistance throughout the range of motion, ${ }^{8,16-18}$ which could be advantageous when training athletes from sports (eg, baseball, tennis) that place increased stress and strain on the shoulder joint.

Although previous research has demonstrated increased velocities when performing the bench press with weighted chains,${ }^{20}$ results from intervention studies in which AR methods were used have been inconclusive. No group differences were found in posttesting of maximal strength following a 7-week training program in which collegiate football players bench-pressed with either elastic bands or weighted chains. ${ }^{11}$ McCurdy et $\mathrm{al}^{8}$ reported similar findings to the aforementioned study, ${ }^{11}$ following a 9 -week training program using chain bench press, in baseball athletes. Conversely, significant gains in maximal upper body strength were observed in collegiate basketball, hockey, and wrestling athletes after a 7-week training program of elastic band bench press compared with one of plate-loaded barbell bench. ${ }^{10}$

At present, training with CAT methods appears to increase maximal strength, ${ }^{15,19}$ while the effects of including
AR techniques in the training programs of athletes are indeterminate..$^{8,10,11}$ Additionally, there is a paucity of research in which CAT and AR training methods have been combined in a resistance training program, yet this type of resistance exercise programming is commonly accepted and employed by practitioners. Previous research has clearly demonstrated that regular resistance exercise will increase muscular strength,,$^{1,2,8-12}$ but the optimal training period and method for such increases has not been elucidated. Therefore, the purpose of the current study was to examine the effect of a 5-week resistance training program in which CAT was used in conjunction with the AR techniques of elastic bands and weighted chains, to determine the effect on maximal upper body strength in collegiate baseball athletes.

\section{Material and methods}

\section{Experimental approach to the problem}

This study was designed to determine the impact of the inclusion of an AR elastic band ("band") or AR weightedchain ("chain") CAT phase, on maximal upper body strength, during an off-season strength and conditioning program for collegiate baseball. Subjects were matched following a maximal effort (1-repetition maximum [1-RM]) bench press test, then were randomly assigned into a band CAT group or a chain CAT group. The athletes were under the direct supervision of a Certified Strength and Conditioning Specialist (CSCS) while they participated in a 5-week training phase that including bench-pressing twice per week. The 5-week band CAT or chain CAT phase was part of the larger 14-week off-season training program and occurred during weeks 6-10. The athletes were experienced lifters who were familiar with the resistance training exercises included in the program and the maximal strength tests that were administered during the testing sessions. Upper body strength was measured by 1-RM bench press testing "pre" (week 5 of 14) and "post" (week 11 of 14) intervention. Adequate recovery time was provided between warm-up sets and maximal attempts.

\section{Subjects}

A total of 24 National Collegiate Athletic Association (NCAA) collegiate baseball players who were familiar with the current strength and conditioning program and had a minimum of 1 year of formal collegiate strength and conditioning experience volunteered to participate in this study. None of the men had participated in band CAT or chain CAT training before this study. All were medically cleared for Intercollegiate Athletic participation and completed a medical history form; all had the risks and benefits explained 
to them beforehand and signed an institutionally approved consent form to participate. The George Mason University Institutional Review Board for Human Subjects approved all study procedures. Sustaining a musculoskeletal injury of the upper body or spine that prevented sports participation within 6 months prior to the study was grounds for exclusion from the study. Prior to strength testing, body height (Seca Stadiometer; Seca GmBH, Hamburg, Germany), and body mass and body composition (Tanita BC-418; Tanita Corporation of America Inc., Arlington Heights, IL, USA) were determined. Subjects were instructed to refrain from upper body exercise for 48 hours prior to each training or testing session. In an effort to maintain consistency across testing conditions, each subject was asked to consume his own identical diet for 24 hours prior to the pre- and posttesting sessions and to consume nothing but water (as needed) for 2 hours prior to the two testing sessions. Training and testing sessions for all subjects were conducted under the supervision of a CSCS, during the off-season training period.

\section{Procedures}

\section{I-RM maximum strength}

For evaluation, 1-RM bench press testing was conducted twice, to determine upper body strength. Pretesting occurred 1 week before the start of the 5-week band CAT or chain CAT program, in what was week 5 of the 14-week off-season training program. Posttesting took place following completion of the 5-week training phase, in what was week 11 of the 14-week off-season training program. Before beginning testing, the athletes completed a specific dynamic flexibility warm-up, which they were familiar with from off-season strength and conditioning training. Next, athletes completed a supervised (by the CSCS) warm-up before testing, to ensure that the intervention exercises were performed correctly. Standard weight lifting power racks (Power Lift ${ }^{\mathbb{}}$; Conner Athletic Products, Inc., Jefferson, IA, USA) were used for the 1-RM tests. The athletes took a timed rest of 3 minutes between maximal attempts. Weight was increased based upon the performance of the previous attempt, and the athlete continued to perform maximal effort attempts until failure or until it was determined the lifting form was compromised. After two failures, testing was stopped, and the best lift was recorded.

\section{Off-season strength and conditioning training program}

The athletes followed a 5-week band CAT or chain CAT bench press training phase that was included as part of the larger 14-week off-season strength and conditioning program designed and implemented by the CSCS who was assigned to baseball. Each group (ie, the band CAT and chain CAT groups) followed the same "periodized", strength training program. All the athlete participants trained together as a team, in the varsity weight room during the baseball offseason (September-December). Specifically, the athletes performed two whole-body lifting sessions, separated by 48 hours, per week for a total of 10 sessions over the 5-week training phase. Training sessions occurred at the same time and on the same days each week. To remain in the off-season program, the athletes were required to make up any missed workouts under the direction of their CSCS within 1 week of being absent. No workouts were missed during the 5-week band CAT or chain CAT training phase. Athletes were asked to notify their CSCS immediately of any concerns, discomfort, pain, or injury related to the training methods. No such issues were raised.

Weight selection for the weekly workouts was based upon percentages from pretesting. Bench pressing occurred during both weekly lifting sessions. Day 1 (CAT maximal effort day) incorporated the periodized protocol for the bench press based on the previously determined 1-RM bench press, and the training intensities ranged from 43\%-97\% 1-RM. The training intensity for day 2 (band CAT or chain CAT) remained at 50\% 1-RM for the 5-week phase. All subjects completed a total of five CAT maximal-effort and five band CAT or chain CAT bench press workouts during the training period. On both days, verbal instructions were given following the eccentric phase of each bench press repetition "to move the weight as fast as possible". Following the warm-up, the workout exercise order progressed from larger muscle groups to smaller muscle groups. The exercise selection and program design characteristics are listed in Table 1.

\section{The accommodating resistance and compensatory acceleration training methods}

Following the 1-RM bench pretest, athletes were matched according to their strength scores, then randomly assigned to either the band CAT or chain CAT group. During the second bench day of each week, bands (Jump Stretch, Inc., Youngstown, OH, USA) and chains (EliteFTS, Inc., London, $\mathrm{OH}, \mathrm{USA}$ ) were attached to the bench press apparatus in a similar manner to what has been described previously. ${ }^{8,10,11}$ The appropriate number of training chains (10 lb) and support chains $(2 \mathrm{lb}$ ) were equally distributed across both ends of the barbell to represent a total load of 50\% 1-RM for chain CAT training. A similar procedure was followed for 
Table I Off-season strength and conditioning program 5-week CAT and AR phase

\begin{tabular}{|c|c|}
\hline & $\begin{array}{l}\text { Volume and intensity } \\
(\text { set } \times \text { rep) at }(\% I-R M)\end{array}$ \\
\hline \multicolumn{2}{|l|}{ Day I } \\
\hline Core warm-up & 10 minutes \\
\hline \multirow[t]{2}{*}{ Olympic lift } & $3 \times 4-6$ reps \\
\hline & Range: $40 \%-80 \%$ \\
\hline CAT maximal & Wk I: I $\times 8$ reps at $43 \%, I \times 6$ reps at $58 \%$, \\
\hline \multirow[t]{9}{*}{ effort bench } & $I \times 5$ reps at $7 \mid \%$, and $2 \times 5$ reps at $85 \%$ \\
\hline & Wk 2 : $I \times 7$ reps at $45 \%, I \times 5$ reps at $60 \%$, \\
\hline & I $\times 4$ reps at $75 \%$, and $2 \times 4$ reps at $85 \%$ \\
\hline & Wk 3: $1 \times 6$ reps at $48 \%, 1 \times 5$ reps at $63 \%$, \\
\hline & I $\times 3$ reps at $76 \%, 2 \times 3$ reps at $90 \%$ \\
\hline & Wk $4: I \times 6$ reps at $50 \%, I \times 5$ reps at $65 \%$, \\
\hline & $1 \times 3$ reps at $79 \%, 2 \times 2$ reps at $93 \%$ \\
\hline & Wk 5: $1 \times 5$ reps at $53 \%, I \times 4$ reps at $68 \%$, \\
\hline & $\mid \times 3$ reps at $81 \%$, and $2 \times 1$ rep at $97 \%$ \\
\hline \multirow[t]{3}{*}{ UB push } & Wk I-2: $2 \times 10$ reps \\
\hline & Wk 3-4: $2 \times 8$ reps \\
\hline & Wk 5: $2 \times 6$ reps \\
\hline Pair exercises & Wk I-2: $3 \times 8$ reps $/ 3 \times 12$ reps \\
\hline UB pull/ & Wk 3-4: $3 \times 6$ reps $/ 3 \times 10$ reps \\
\hline LB pull & Wk 5: $3 \times 6$ reps $/ 3 \times 8$ reps \\
\hline \multicolumn{2}{|l|}{ Pair exercises } \\
\hline UB push/ & Wk I-3: $2 \times 5$ reps $/ 2 \times 10$ reps \\
\hline LB push & Wk 4-5: $2 \times 6$ reps $/ 2 \times 8$ reps \\
\hline Shoulder & $3 \times 12$ reps \\
\hline prehabilitation & 3 exercises \\
\hline \multicolumn{2}{|l|}{ Day 2} \\
\hline Core warm-up & 10 minutes \\
\hline \multirow[t]{2}{*}{ Olympic Lift } & $3 \times 4-6$ reps \\
\hline & Range: $30 \%-60 \%$ \\
\hline $\begin{array}{l}\text { AR bench (band CAT } \\
\text { or chain CAT) }\end{array}$ & Wk I-5: $9 \times 3$ reps of all sets at $50 \%$ I-RM \\
\hline \multirow[t]{3}{*}{ LB push } & Wk I-2: $3 \times 12$ reps \\
\hline & Wk 3-4: $3 \times 10$ reps \\
\hline & Wk 5: $3 \times 8$ reps \\
\hline Pair exercises & Wk I-2: $3 \times 12$ reps both exercises \\
\hline LB push/ & Wk 3-4: $3 \times 10$ reps both exercises \\
\hline UB pull & Wk 5: $3 \times 8$ reps both \\
\hline \multicolumn{2}{|l|}{ Pair exercises } \\
\hline UB pull/ & Wk I-3: $2 \times 10$ reps $/ 2 \times 8$ reps \\
\hline LB pull & Wk 4-5: $2 \times 8 \mathrm{reps} / 2 \times 6$ reps \\
\hline Shoulder & $1 \times 20-30$ seconds \\
\hline Circuit & 4 exercises \\
\hline
\end{tabular}

Note: Allow $\leq 90$ seconds rest between paired exercises.

Abbreviations: I-RM, I-repetition maximum; AR, accommodating resistance; CAT, compensatory acceleration training; LB, lower body; rep, repetition; UB, upper body; Wk, week.

the band CAT training, with bands attached whose maximal resistance when extended, plus the barbell, represented a total load of each individual subject's 50\% 1-RM. The amount of band resistance was determined according to the equipment company's specifications. Both band CAT and chain CAT groups performed nine sets of three repetitions at 50\% 1-RM, with instructions to move the weight as quickly as possible.
The bar grips consisted of close grip (hands 14 inches apart) for sets 1-3, midgrip (hands 19 inches apart) for sets 4-6, and wide grip (hands 24 inches apart) for sets 7-9. The rest period between each bench set of the band CAT or chain CAT workout was 45 seconds.

\section{Statistical analyses}

Descriptive statistics were computed for each of the variables. Independent group $t$-tests were conducted on the characteristics of the subjects. To evaluate changes from pretest to posttest in the dependent variable, a $2 \times 2$ mixed factorial analysis of variance was calculated to compare the dependent variable (Bench Press) across groups (band CAT and chain CAT) and testing periods ("pre" and "post"). The alpha level was set at 0.05 . All the statistical procedures were conducted using the Statistical Package for the Social Sciences (SPSS Statistics for Windows, Version 20.0; IBM Corp, Armonk, NY, USA).

\section{Results}

The primary purpose of this research was to determine whether differences existed between maximal upper body strength following inclusion of 5 weeks of band CAT or chain CAT into an off-season strength and conditioning programming, for a group of collegiate baseball players. The goal was to determine which method (band CAT or chain CAT) would produce greater increases in upper body strength over the 5-week training period.

All data are presented as means \pm standard error of the mean. Twenty-four subjects completed the study. Their physical characteristics are recorded in Table 2. Subjects were matched according to their pretest bench press scores, then randomly assigned into the band CAT or chain CAT group. Independent group $t$-tests were completed to determine whether there were differences in physical characteristics between the band CAT and chain CAT groups. A significant difference existed $(P=0.02)$ in body fat percentage between

Table 2 Physical characteristics of subjects

\begin{tabular}{lcc}
\hline Item & $\begin{array}{l}\text { Chain CAT } \\
(\mathbf{n}=\mathbf{I} \mathbf{2})\end{array}$ & $\begin{array}{l}\text { Band CAT } \\
(\mathbf{n}=\mathbf{I} \mathbf{2})\end{array}$ \\
\hline Age (years) & $20 \pm 1.0$ & $20.3 \pm 1.0$ \\
Height $(\mathrm{cm})$ & $180.1 \pm 8.4$ & $181.4 \pm 6.7$ \\
Weight $(\mathrm{kg})$ & $85.2 \pm 3.8$ & $88.9 \pm 4.0$ \\
\% body fat & $10.7 \pm 1.6$ & $13.9 \pm 1.9^{\mathrm{a}}$ \\
Pretest I-RM bench press $(\mathrm{kg})$ & $99.6 \pm 5.8$ & $101.7 \pm 7.6$ \\
\hline
\end{tabular}

Notes: Values are expressed as mean $\pm \mathrm{SEM}$; ${ }^{\mathrm{P}}=0.02$.

Abbreviations: I-RM, I-repetition maximum; CAT, compensatory acceleration training; Chain CAT, chain bench press group; Band CAT, band bench press group; SEM, standard error of the mean. 


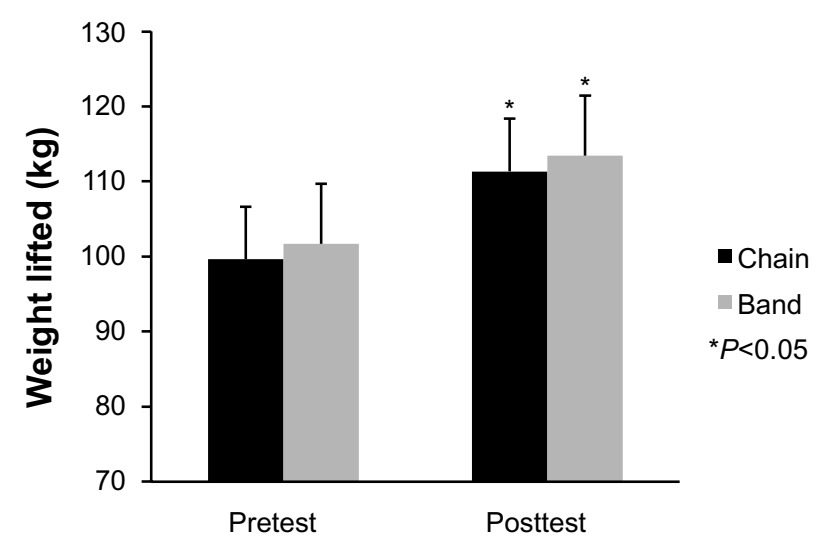

Figure I Pre- and posttest values of I-RM bench press (kg). Abbreviation: I-RM, I-repetition maximum.

the band CAT $(13.0 \% \pm 1.9 \%)$ and chain CAT $(10.7 \% \pm 1.6 \%)$ groups.

\section{Strength testing}

The descriptive statistics for pre- and posttests of 1-RM bench press are presented in Figure 1. There was no interaction effect between group and time $\left(F_{1,22}=0.00, P=1.00\right)$. No significant difference $\left(F_{1,22}=0.04, P=0.84\right)$ existed between the band CAT and chain CAT groups. A significant difference $\left(F_{1,22}=88.46, P=0.001\right)$ was observed between pretest $(100.7 \pm 4.7 \mathrm{~kg})$ and posttest $(112.4 \pm 5.4 \mathrm{~kg})$ of $1-\mathrm{RM}$ bench as a result of the 5-week training phase. The band CAT and chain CAT groups increased their maximal bench press by $11.74 \mathrm{~kg}$ and $11.75 \mathrm{~kg}$, respectively.

\section{Discussion}

This is the first study designed to examine differences in training using the AR methods of weighted chains and elastic bands while employing CAT lifting techniques in resistancetrained baseball athletes. The overall findings supported no difference between the two AR methods of upper body exercise. Results from previous research using these AR methods without CAT have been inconclusive. ${ }^{8,10}$

We observed that maximal upper body strength gains were similar between 5 weeks of band CAT and chain CAT training. Baseball athletes in both groups experienced a statistically significant increase in their maximal bench press from pre- to posttesting, with the band CAT group increasing $11.6 \%$ (ie, $11.74 \mathrm{~kg}$ ) and the chain-CAT group increasing $11.8 \%$ (ie, $11.75 \mathrm{~kg}$ ). These findings are consistent with previous research in which 7 weeks of AR training in football players produced an increase in upper body strength, with no group difference between elastic bands $(+7.8 \%)$ and weighted chains $(+7 \%) .{ }^{11}$ Similarly, a 9-week AR intervention study, without the use of CAT, in baseball athletes resulted in increased upper body strength, with no group difference between weighted chains $(+14.7 \%)$ or plate-loaded barbells $(+6.3 \%){ }^{8}$ On the other hand, 7 weeks of AR training with elastic bands, without the use of CAT, produced a significant group difference in upper body strength $(+8 \%)$ compared with plate-loaded barbell training $(+4 \%) .{ }^{10}$

Although long considered to be a popular training method in the powerlifting community, ${ }^{16-18}$ there remains a dearth of published intervention studies on upper body strength and CAT. One intervention study used CAT techniques, without AR, for a 14-week off-season strength-training program with football athletes. ${ }^{15}$ Significant group differences were observed, as the athletes training with CAT experienced a 9.4\% increase in maximal bench press compared with a $3.8 \%$ increase in the control group. ${ }^{15}$

It is unclear whether or not the increases in upper body strength in the present study were a result of the band CAT and chain CAT training methods or merely adaptations occurring as a result of consistent resistance training during the off-season. However, it is of interest to note that the upper body strength gains in the 5-week study are of a larger magnitude than those previously reported for longer training periods with football athletes. ${ }^{22,23} \mathrm{~A}$ linear periodized, 15-week off-season program for football athletes increased maximal bench press values by $7.8 \%,{ }^{22}$ while a 10 -week planned nonlinear periodization program increased maximal bench press values by $4 \%$ in a similar subject population. ${ }^{23}$ This finding from the current study is noteworthy because it indicates that combining AR methods with CAT techniques may result in a more rapid gain in maximal upper body strength $(\sim 12 \%)$ than has been previously reported. A consistent goal of strength and conditioning professionals is to maximize results in minimum time; ${ }^{24}$ therefore, they may find it beneficial to have athletes train using CAT in conjunction with AR for designated phases of the off-season strength and conditioning program. However, caution should be exercised in setting specific expectations for strength gains. Improvement may vary across sport, level of athlete, and length of the training phase. Further research is necessary to compare these training methods in more detail.

Resistance training is an accepted part of off-season training for baseball, with the primary goals being muscle strengthening ${ }^{2,3,6}$ and injury prevention. ${ }^{25}$ Shoulder injuries are common in baseball, due to the high volume of throwing, and while the bench press is generally considered to be an optimal exercise for developing upper body musculature, it has been reported to increase stress on the shoulder, 
particularly at the end of the eccentric phase..$^{26,27}$ Performing the bench press with weighted chains is thought to minimize stress, due to the variable resistance throughout the range of motion. ${ }^{8,16-18}$ Published research ${ }^{8,10,11}$ as well as publications from the popular press ${ }^{16-18}$ caution against the risk of increased soreness when training with elastic bands. While soreness was not measured in the current study, athletes were instructed to report pain, injury, or increased soreness to the investigator. Neither the band CAT nor the chain CAT groups reported any incidence of the aforementioned symptoms.

We acknowledge some study limitations. First, the 24 collegiate baseball athletes had $\geq 1$ year of formal collegiate strength training experience. Published research has shown that strength gains are affected by the type of training program and previous training experience. ${ }^{12,23,28}$ Future study that addresses the relationship between training experience and upper body strength in connection with AR and CAT training methods is warranted. Second, the absence of a control group poses a limitation. Findings from previous AR training studies established significant upper body strength gains with elastic bands compared with plate-loaded barbells, ${ }^{10,28}$ yet in other studies, weighted chains failed to produce a greater strength gain than either elastic band ${ }^{11}$ or plate-loaded barbell training. ${ }^{8}$ Due to the limited number of trained baseball athletes available (ie, 24), a conscious decision was made to include only the two intervention groups - band CAT and chain CAT. Having a larger sample size would have been more conducive to the inclusion of a control group. Finally, the AR-CAT training was included as a 5-week training phase within the off-season strength and conditioning program as a whole, and the subjects were trained baseball athletes who were following sport-specific training regimens and involved in regular activities with definite neuromuscular demands. Thus, results may vary in untrained individuals or in athletes from other sports. Future research is needed to examine what role the training volume and intensities of other program exercises used in conjunction with CAT and AR methods may play in the upper body strength adaptations. Additionally, future examination of muscle activity patterns across these training methods may help to ascertain potential mechanisms of adaptation.

\section{Conclusion}

The results of this study suggest the following in trained collegiate baseball athletes:

1. The use of band CAT or chain CAT methods will increase maximal upper body strength.
2. AR and CAT can be implemented together as an effective training alternative for a 5-week phase during off-season programming.

3. The use of band CAT or chain CAT appears to be a viable, short-term training alternative since no concerns were expressed or injuries sustained.

\section{Acknowledgments}

The author would like to thank the athletic trainers, coaches, and student-athletes from the intercollegiate baseball program, for their contribution to the study.

\section{Disclosure}

The author reports no conflicts of interest in this work.

\section{References}

1. Lachowetz T, Evon J, Pastiglione J. The effect of an upper body strength program on intercollegiate baseball throwing velocity. J Strength Cond Res. 1998;12(2):116-119.

2. Escamilla RF, Ionno M, deMahy MS, et al. Comparison of three baseballspecific 6-week training programs on throwing velocity in high school baseball players. J Strength Cond Res. 2012;26(7):1767-1781.

3. Miyaguchi K, Demura S. Relationship between upper-body strength and bat swing speed in high-school baseball players. J Strength Cond Res. 2012;26(7):1786-1791.

4. Spaniol FJ. Baseball Athletic Test: a baseball-specific test battery. Strength Cond J. 2009;31(2):26-29.

5. Kohmura Y, Aoki K, Yoshigi H, Sakuraba K, Yanagiya T. Development of a baseball-specific battery of tests and a testing protocol for college baseball players. J Strength Cond Res. 2008;22(4):1051-1058.

6. Mangine GT, Hoffman JR, Fragala MS, et al. Effect of age on anthropometric and physical performance measures in professional baseball players. J Strength Cond Res. 2013;27(2):375-381.

7. Hoffman JR, Vazquez J, Pichardo N, Tenenbaum G. Anthropometric and performance comparisons in professional baseball players. J Strength Cond Res. 2009;23(8):2173-2178.

8. McCurdy K, Langford G, Ernest J, Jenkerson D, Doscher M. Comparison of chain- and plate-loaded bench press training on strength, joint pain, and muscle soreness in Division II baseball players. J Strength Cond Res. 2009;23(1):187-195.

9. Szymanski DJ, Szymanski JM, Bradford TJ, Schade RL, Pascoe DD. Effect of twelve weeks of medicine ball training on high school baseball players. J Strength Cond Res. 2007;21(3):894-901.

10. Anderson CE, Sforzo GA, Sigg JA. The effects of combining elastic and free weight resistance on strength and power in athletes. J Strength Cond Res. 2008;22(2):567-574.

11. Ghigiarelli JJ, Nagle EF, Gross FL, Robertson RJ, Irrgang JJ, Myslinski T. The effects of a 7-week heavy elastic band and weight chain program on upper-body strength and upper-body power in a sample of division 1-AA football players. $J$ Strength Cond Res. 2009;23(3):756-764.

12. Kraemer WJ, Ratamess NA. Fundamentals of resistance training: progression and exercise prescription. Med Sci Sports Exerc. 2004;36(4):674-688.

13. Behm DG, Sale DG. Intended rather than actual movement velocity determines velocity-specific training response. J Appl Physiol (1985). 1993;74(1):359-368.

14. Swinton PA, Lloyd R, Agouris I, Stewart A. Contemporary training practices in elite British powerlifters: survey results from an international competition. J Strength Cond Res. 2009;23(2):380-384. 
15. Jones K, Hunter G, Fleisig G, Escamilla R, Lemak L. The effects of compensatory acceleration on upper-body strength and power in collegiate football players. J Strength Cond Res. 1999;13(2):99-105.

16. westside-barbell.com [homepage on the Internet]. Chain reaction: Accommodating leverages. Westside Barbell; 2003 [updated June 14, 2013; cited April 10, 2014]. Available from: http://www.westsidebarbell.com/index.php/articles-by-louie-simmons/articles-published-in2003/355-chain-reaction-accommodating-leverages. Accessed June 17, 2014.

17. westside-barbell.com [homepage on the Internet]. Chains and bands. Westside Barbell; 2004 [updated June 15, 2013; cited April 11, 2014]. Available from: http://www.westside-barbell.com/index.php/articlesby-louie-simmons/articles-published-in-2004/367-chains-and-bands. Accessed June 17, 2014.

18. westside-barbell.com [homepage on the Internet]. Training methods part 1: Speed day. Westside Barbell; 2004 [updated June 14, 2013; cited April 11, 2014]. Available from: http://www.westside-barbell. com/index.php/articles-by-louie-simmons/articles-published-in-2004 /359-training-methods-part-1-speed-day. Accessed June 17, 2014.

19. Jones K, Bishop P, Hunter G, Fleisig G. The effects of varying resistance-training loads on intermediate- and high-velocity-specific adaptations. J Strength Cond Res. 2001;15(3):349-356.

20. Baker DG, Newton RU. Effect of kinetically altering a repetition via the use of chain resistance on velocity during the bench press. $J$ Strength Cond Res. 2009;23(7):1941-1946.
21. Siff MC. Supertraining. 6th ed. Denver, CO: Supertraining Institute, 2003.

22. Hoffman JR, Ratamess NA, Klatt M, et al. Comparison between different off-season resistance training programs in Division III American college football players. J Strength Cond Res. 2009;23(1):11-19.

23. Smith RA, Martin GJ, Szivak TK, et al. The effects of resistance training prioritization in NCAA Division I Football summer training. J Strength Cond Res. 2014;28(1):14-22.

24. Jones MT, Parker BM, Cortes N. The effect of whole-body vibration training and conventional strength training on performance measures in female athletes. J Strength Cond Res. 2011;25(9): 2434-2441.

25. Lachowetz T, Drury D, Elliot R, Evon J, Pastiglione J. The effect of an intercollegiate baseball strength program on the reduction of shoulder and elbow pain. J Strength Cond Res. 1998;12(1):46-51.

26. Durall CJ, Manske RC, Davies GJ. Avoiding shoulder injury from resistance training. Strength Cond J. 2001;23(5):10-18.

27. Fees M, Decker T, Snyder-Mackler L, Axe MJ. Upper extremity weighttraining modifications for the injured athlete. A clinical perspective. Am J Sports Med. 1998;26(5):732-742.

28. Bellar DM, Muller MD, Barkley JE, et al. The effects of combined elastic- and free-weight tension vs free-weight tension on one-repetition maximum strength in the bench press. J Strength Cond Res. 2011;25(2): 459-463.
Open Access Journal of Sports Medicine

\section{Publish your work in this journal}

Open Access Journal of Sports Medicine is an international, peer-reviewed, open access journal publishing original research, reports, reviews and commentaries on all areas of sports medicine. The manuscript management system is completely online and includes a very quick and fair peer-review system.

\section{Dovepress}

Visit http://www.dovepress.com/testimonials.php to read real quotes from published authors. 